https://doi.org/10.23913/ride.v12i24.1123

Artículos científicos

\title{
Caracterización de los factores docentes en torno al índice de reprobación en universitarios
}

Characterization of the Teaching Factors Around the Reprobation Rate in

University

Caracterização dos fatores de ensino em torno da taxa de reprovação em estudantes universitários

Ángel Esteban Torres-Zapata

Universidad Autónoma del Carmen, México

etorre@pampano.unacar.mx https://orcid.org/0000-0002-6066-3258

Alejandra Karina Pérez-Jaimes Universidad Autónoma del Estado de México, México

Grupo Colegio Mexiquense, México akperezj001@profesor.uaemex.mx https://orcid.org/0000-0003-3079-1782

Carmen Cecilia Lara-Gamboa Universidad Autónoma de Campeche, México carclara@uacam.mx https://orcid.org/0000-0001-7893-9913

César Uziel Estrada-Reyes Universidad Mexiquense del Bicentenario, México Grupo Colegio Mexiquense, México cesar.estrada.Iic@colmexuni.edu.mx https://orcid.org/ 0000-0003-4932-2214 


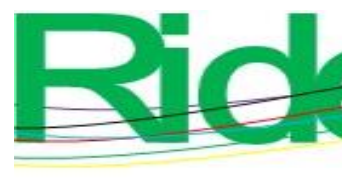

Revista Iberoamericana para la Investigación y el Desarrollo Educativo ISSN $2007-7467$

\section{Resumen}

El objetivo del presente ejercicio de investigación fue caracterizar los factores docentes que inciden en la reprobación de estudiantes universitarios. El estudio tuvo un enfoque cuantitativo no experimental transversal. La selección de la población de estudio fue no probabilística. Se incluyó a la totalidad de los profesores que impartieron las unidades de aprendizaje durante los ciclos escolares febrero-junio 2019 y agosto-diciembre 2019 en una universidad de México. Se llevó a cabo un análisis descriptivo, a través del cual se determinaron la media, la mediana, las frecuencias absolutas y la desviación estándar. Además, se vincularon los factores docentes con el índice de reprobación a partir de una prueba de ji al cuadrado. Entre los resultados destaca que el índice de reprobación del periodo de análisis de los cursos de competencia genérica fue de $34.37 \%$, de los interdisciplinarios $10.85 \%$ y de los de competencias específicas de $16.03 \%$. Asimismo, $68.97 \%$ de los profesores demostró un nivel máximo de estudios de maestría y 88.89 \% refirió más de cinco años de experiencia profesional en el ámbito docente. $\mathrm{Al}$ asociar el índice de reprobación con las variables de estudio, no hubo significatividad con las unidades de aprendizaje por competencia y la evaluación docente; sin embargo, sí hubo significancia con el nivel máximo de estudios, con la experiencia profesional y con la preparación pedagógica.

Palabras clave: abandono escolar, docentes, reprobación.

\section{Abstract}

The objective of this research exercise was to characterize the teaching factors that influence the failure of university students. The study had a quantitative non-experimental crosssectional approach. The selection of the study population was non-probabilistic. All the teachers who taught the learning units during the February-June 2019 and August-December 2019 school cycles at a university in Mexico were included. A descriptive analysis was carried out, through which the statistical mean, median, absolute frequencies and standard deviation were determined. In addition, the teaching factors were linked to the failure rate from a chi-square test. Among the results, it stands out that the failure rate for generic competency courses was $34.37 \%, 10.85 \%$ for interdisciplinary courses, and $16.03 \%$ for specific competences. Likewise, $68.97 \%$ of the teachers demonstrated a maximum level of master's studies and $88.89 \%$ referred more than five years of professional experience in the teaching field. When associating the failure rate with the study variables, there was no 


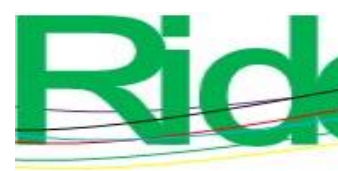

Revista Iberoamericana para la
Investigación y el Desarrollo Educativo
ISSN $2007-7467$

significance with the learning units by competence and teacher evaluation; however, there was significance with the maximum level of studies, with professional experience and with pedagogical preparation.

Keywords: school dropout, teachers, school failure.

\section{Resumo}

O objetivo deste exercício de pesquisa foi caracterizar os fatores de ensino que influenciam o fracasso de estudantes universitários. O estudo teve uma abordagem quantitativa não experimental de corte transversal. A seleção da população de estudo foi não probabilística. Foram incluídos todos os professores que lecionaram nas unidades de aprendizagem durante os ciclos escolares de fevereiro a junho de 2019 e agosto a dezembro de 2019 em uma universidade no México. Foi realizada análise descritiva, por meio da qual foram determinadas a média, mediana, frequências absolutas e desvio padrão. Além disso, os fatores de ensino foram associados à taxa de reprovação por meio de um teste de quiquadrado. Dentre os resultados, destaca-se que a taxa de reprovação do período de análise dos cursos de competência genérica foi de 34,37\%, dos interdisciplinares $10,85 \%$ e das competências específicas 16,03\%. Da mesma forma, 68,97\% dos professores demonstraram nível máximo de estudos de mestrado e 88,89\% referiram mais de cinco anos de experiência profissional na área de docência. Ao associar a taxa de reprovação com as variáveis do estudo, não houve significância com as unidades de aprendizagem por competência e avaliação do professor; Porém, houve significância com o nível máximo de estudos, com experiência profissional e com preparação pedagógica.

Palavras-chave: evasão escolar, professores, fracasso.

Fecha Recepción: Mayo 2021

Fecha Aceptación: Noviembre 2021

\section{Introducción}

México enfrenta grandes retos en cuanto al acceso y permanencia de los alumnos en las instituciones educativas del nivel superior. El índice de abandono durante el ciclo 20192020 fue de $7.4 \%$ del total de los estudiantes, mientras que el porcentaje de absorción fue de 15.7 \% (Secretaría de Educación Pública [SEP], 2020). La principal problemática es la calidad del sistema educativo. 


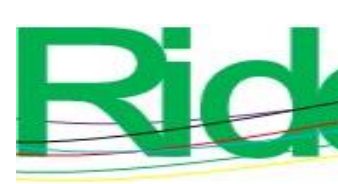

Revista Iberoamericana para la Investigación y el Desarrollo Educativo ISSN 2007-7467

y Pérez, 2014). Esto implica que el docente debe involucrarse en una capacitación y formación profesional constante y profunda, así podrá cumplir con su rol de agente transformador de la sociedad. Sin duda, es fundamental el desarrollo humano, personal y social de este actor (Nieva y Martínez, 2016).

Una vez que se localiza al docente como punto focal surgen las siguientes interrogantes: ¿cuál es el factor que no permite el correcto desarrollo de la enseñanza aprendizaje?, ¿la preparación profesional o pedagógica será pieza clave?, ¿cuál es el factor que más se debe cuidar en la práctica de la docencia? (Pérez, Estrada y Estrada, 2021). El supuesto de que un buen docente es el que logra que los estudiantes alcancen los conocimientos necesarios para acreditar su materia podría llegar a sugerir de manera errónea que no es necesario evaluar el trabajo o desempeño de cada maestro, puesto que este pudiera inferirse a partir del rendimiento de sus alumnos (Martínez, 2013).

Por lo anterior, se plantea como objetivo general de la investigación caracterizar los factores docentes que inciden en la reprobación de estudiantes universitarios y generar información útil para la toma de decisiones en la actualización del programa educativo.

\section{Materiales y métodos}

Estudio de tipo cuantitativo, observacional, descriptivo, no experimental, de corte transversal. La población estuvo conformada por 29 profesores que impartieron las unidades de aprendizaje durante los ciclos escolares febrero-junio 2019 y agosto-diciembre 2019 en el programa educativo del área de la salud de una universidad mexicana. Fueron excluidos de la investigación aquellos profesores que, si bien impartieron alguna asignatura en los interperiodos, no lo hicieron en los ciclos escolares contemplados en el estudio. Los factores que se relacionaron con el índice de reprobación fueron los siguientes:

- $\quad$ Tipo de materias por competencia (genéricas, interdisciplinarias y específicas [básicas de la carrera, profesionalizantes, terminal obligatorio y terminal optativo]).

- $\quad$ Máximo grado de estudios del docente (licenciatura, maestría y doctorado).

- $\quad$ Experiencia profesional docente (uno a dos años de experiencia, entre dos y cinco años de experiencia y con más de cinco años de experiencia docente).

- Preparación pedagógica (un curso pedagógico tomado, de uno a tres cursos tomados y más de cuatro cursos pedagógicos tomados). 


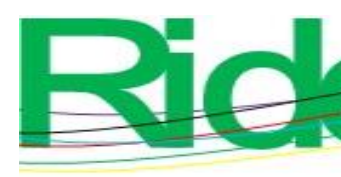

Revista Iberoamericana para la
Investigación y el Desarrollo Educativo
ISSN $2007-7467$

- Calificación del desempeño docente otorgado por el estudiante (excelente [9.5-10], bueno [8.9-9.4], regular [8.3- 8.8] y bajo [7-8.2]).

Para identificar el índice de reprobación, se evaluaron las calificaciones obtenidas por los estudiantes, contenidas en los programas institucionales de la trayectoria escolar, y se clasificaron como "Aprobados" y "No aprobados". Todas las variables fueron suministradas como datos por los sistemas informáticos institucionales, proporcionados por la coordinación del programa educativo de la institución de estudio. Se solicitó de manera formal y por escrito la autorización para la puesta en marcha del estudio al coordinador y a los líderes de las academias del programa educativo. Igualmente, al descartar conflictos de interés, se valoró positivamente la propuesta por el grupo disciplinar adscrito al programa. Se contó con la participación de investigadores externos a la institución estudiada. Se utilizó como herramienta de asistencia estadística el paquete SPSS versión 25.0 para Windows. Como significancia estadística se tomó el valor de $p \leq 0.05$. Se generó un análisis descriptivo, a través del cual se obtuvieron medias, medianas, frecuencias absolutas y medidas de dispersión. Por último, con la prueba ji al cuadrado se relacionaron los factores docentes y el índice de reprobación.

\section{Resultados}

Los 29 profesores que conformaron la población de estudio impartieron al menos una unidad de aprendizaje durante los semestres del 2019 en el programa educativo de referencia. En cuanto al género, $79.31 \%$ (23) de la planta académica incluida en la investigación es de género femenino y $20.69 \%$ (seis) masculino.

El modelo educativo declarado es por competencias, centrado en el proceso de aprendizaje significativo del estudiante. Respecto a la estructura curricular del programa educativo estudiado, se promueven las competencias de tipo genéricas, interdisciplinarias y específicas. La tabla 1 muestra el índice de reprobación por competencia. 
Tabla 1. Índice de reprobación por área de competencia

\begin{tabular}{|l|c|r|r|r|r|r|r|r|r|}
\hline \multirow{2}{*}{ Competencias } & Inscritos & \multicolumn{5}{c|}{ Aprobado } & \multicolumn{4}{c|}{ No aprobados } \\
\cline { 3 - 10 } & & $\mathrm{f}$ & $\%$ & Media & $\mathrm{DE}$ & $\mathrm{f}$ & $\%$ & Media & DE \\
\hline Genéricas & 355 & 233 & 66 & 19.72 & 16.61 & 122 & 34.37 & 5.52 & 4.49 \\
\hline Interdisciplinarios & 295 & 263 & 89 & 29.5 & 17.47 & 32 & 10.85 & 3.2 & 2.64 \\
\hline Especificas & 911 & 765 & 84 & 18.21 & 9.7 & 146 & 16.03 & 3.48 & 3.17 \\
\hline $\begin{array}{l}\text { Específicas: } \\
\text { básicos de la carrera }\end{array}$ & 212 & 176 & 83 & 22 & 9.3 & 36 & 16.98 & 4.5 & 3.28 \\
\hline $\begin{array}{l}\text { Específicas: } \\
\text { profesionalizante }\end{array}$ & 579 & 485 & 84 & 17.96 & 9.88 & 94 & 16.23 & 3.48 & 2.96 \\
\hline $\begin{array}{l}\text { Específicas: } \\
\text { terminal obligatorio }\end{array}$ & 65 & 63 & 97 & 12.6 & 2.15 & 2 & 3.08 & 0.4 & 0.48 \\
\hline $\begin{array}{l}\text { Específicas: } \\
\text { terminal optativo }\end{array}$ & 55 & 41 & 75 & 20.5 & 12.5 & 14 & 25.45 & 7 & 3 \\
\hline
\end{tabular}

\section{Fuente: Elaboración propia}

En la tabla 2 se presenta la clasificación de los profesores por unidades de aprendizaje y tipo de competencia.

Tabla 2. Clasificación de los profesores por tipo de curso que imparte

\begin{tabular}{|l|r|r|}
\hline Competencias & $\mathrm{f}$ & $\%$ \\
\hline Genéricas & 4 & 13.79 \\
\hline Interdisciplinarios & 8 & 27.59 \\
\hline Específicas: básicos de la carrera & 3 & 10.34 \\
\hline Específicas: profesionalizante & 10 & 34.48 \\
\hline Específicas: terminal obligatorio & 2 & 6.90 \\
\hline Específicas: terminal optativo & 2 & 6.90 \\
\hline Total & 29 & 100 \\
\hline
\end{tabular}

\section{Fuente: Elaboración propia}

En relación con el nivel máximo de estudios con el que contaban los profesores que impartieron las unidades de aprendizaje, $20.69 \%(n=6)$ tenían licenciatura afín al curso impartido, $68.97 \%(n=20)$ ostentaban grado de maestría y $13.79 \%(n=4)$ de doctorado. 


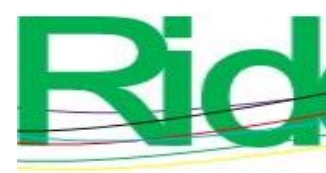

\section{Revista Iberoamericana para la Investigación y el Desarrollo Educativo ISSN 2007 - 7467}

En lo referente a la experiencia profesional docente, $7.71 \%$ (dos) tenía entre uno y dos años de experiencia, $11.11 \%$ (tres) entre dos y cinco años y $88.89 \%$ (24) contaba con más de cinco años.

Respecto a la preparación pedagógica, $100 \%$ contaba con al menos un curso en el área; $6.90 \%(n=2)$ declaró más de cuatro cursos y $93.10 \%(n=27)$ declaró de uno a tres cursos.

Finalmente, en cuanto a los resultados de la evaluación del desempeño docente realizada por 178 estudiantes universitarios durante los ciclos escolares del 2019, el claustro docente obtuvo una media de $9.6 \pm 0.01$, es decir, una calificación de excelente, como se plasma en la tabla 3.

Tabla 3. Evaluación de docentes por los estudiantes

\begin{tabular}{|l|r|r|}
\hline Resultados & $\mathrm{f}$ & $\%$ \\
\hline Excelente (9.5-10) & 27 & 93.10 \\
\hline Bueno (8.9-9.4) & 1 & 3.45 \\
\hline Regular (8.3- 8.8) & 1 & 3.45 \\
\hline Bajo (7-8.2) & 0 & 0 \\
\hline
\end{tabular}

Fuente: Elaboración propia

Mientras que respecto al porcentaje de reprobación, se recurrió a seis categorías que se describen en la tabla 4.

Tabla 4. Clasificación de los docentes por los porcentajes de reprobación

\begin{tabular}{|l|c|r|}
\hline Porcentaje de reprobación & $\mathrm{f}$ & $\%$ \\
\hline $0-10$ & 11 & 37.93 \\
\hline $11-20$ & 6 & 20.69 \\
\hline $21-30$ & 4 & 13.79 \\
\hline $31-40$ & 3 & 10.34 \\
\hline $41-50$ & 3 & 10.34 \\
\hline$>50$ & 2 & 6.90 \\
\hline Totales & 29 & 100 \\
\hline
\end{tabular}

Fuente: Elaboración propia 


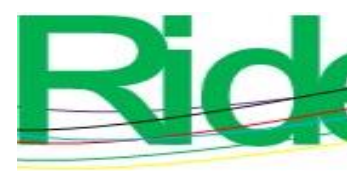

Revista Iberoamericana para la Investigación y el Desarrollo Educativo ISSN 2007 - 7467

Para analizar la asociación del índice de reprobación con las variables de estudio se realizó la prueba estadística de ji al cuadrado. Y se encontró que, con la clasificación de las unidades de aprendizaje por competencia, $p=0.87$, que no es estadísticamente significativo. Respecto a la evaluación docente, se obtuvo un valor de $p=0.54$.

Por otro lado, la variable asociada con el nivel máximo de estudios demostró una relación positiva, con un valor de $p=0.04$, lo cual es estadísticamente significativo, al igual que la experiencia profesional, cuyo valor fue $p=0.03$, y la preparación pedagógica, con $p=0.05$.

\section{Discusión}

En la actualidad, el sistema educativo en México enfrenta como uno de los principales problemas la deserción escolar. El acto de reprobar es un signo de bajo aprovechamiento escolar, que denota desigualdad en el aprendizaje y que se considera como la principal causa del fracaso escolar. Además, como consta en diversos estudios, es un signo fehaciente de una baja calidad educativa. Esto ubica a México entre las naciones con niveles más bajos de rendimiento escolar (Díaz y Ruiz, 2018).

Existen índices de fracaso considerados como normales por los profesores; sería poco creíble que en una institución educativa no exista reprobación. Dado que los puntos de corte para medir los porcentajes de reprobación son relativos, se utilizan las recomendaciones emitidas por organismos evaluadores. Así, el Consejo Nacional para la Calidad de Programas Educativos en Nutriología [Concapren] (2016) estipula como ideales valores inferiores a $10 \%$, mientras que los lineamientos del Programa de Carrera Docente en Universidades Públicas Estatales (UPES) sugieren tasas de aprobación de $70 \%$ o más, esto es, una aceptación de reprobación no mayor a 30 \% (Dirección General de Educación Superior Universitaria, 2020). Un fenómeno considerado hasta cierto punto normal son los índices elevados de reprobación en los primeros ciclos escolares (Ciro y Reyes, 2017); sin embargo, esta es una referencia poco estable, puesto que en un ciclo escolar el mismo profesor puede tener una variabilidad marcada en los porcentajes de reprobación (entre 10 \% y $30 \%$ ) y al siguiente ciclo presentar un aumento o disminución considerable e incluso en el mismo ciclo pero en unidades de aprendizajes diferentes (Villalón, Palma, Medina y Sillero, 2016). La complejidad de los fenómenos en el proceso educativo ha sido señalada por la Organización para la Cooperación y el Desarrollo Económicos [OCDE] (2006), que guarda registros de 


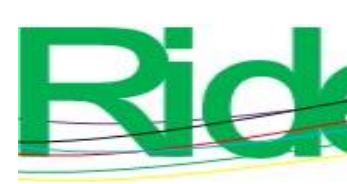

Revista Iberoamericana para la Investigación y el Desarrollo Educativo ISSN 2007 - 7467

cobertura en la educación superior de los países miembros con alcance en $25 \%$ de la matrícula estudiantil, quienes concluyeron la escolaridad del nivel básico y medio superior con posibilidad de transitar a estudios de nivel superior e incluso a un posgrado.

La magnitud del problema se puede trazar con los datos de la tabla 1. Allí se observa que las unidades de aprendizaje con mayor afectación son las genéricas, las cuales son básicas y transversales en las diversas licenciaturas. Las unidades de aprendizaje que integran estas competencias son sistémicas o integradoras, transversales y transferibles e incluyen los cursos de inglés A, B, I, II, II y IV y razonamiento lógico, donde se presenta la mayor afectación. De suma importancia es el reconocimiento de la correlación existente entre la reprobación y la deserción escolar.

Los resultados de la asociación entre la reprobación y el área de competencia no son significativos, sin embargo, se puede observar que la mayor tasa de reprobación se da en las unidades de aprendizaje que contribuyen a competencias genéricas, sobre todo en los niveles de inglés. Estos resultados son equiparables a los vertidos por Sánchez y De Santiago (2017), quienes encontraron que el tiempo insuficiente, según la percepción del estudiante, fue la causa con mayor influencia en la reprobación de inglés. En todas las licenciaturas se observa el mismo fenómeno. Y como segundo factor influyente en la reprobación se señaló a la carencia de buenas estrategias de estudio (Sánchez y De Santiago, 2017).

Por otro lado, pese a que el profesor es reconocido como eje central del aprendizaje universitario, no es sencillo encontrar un consenso sobre el perfil que debe tener un buen docente en el nivel superior (Valerio y Rodríguez, 2017). En cuanto a la formación de los profesores universitarios, los grados académicos están directamente relacionados con el interés de incidir en la función docente de investigación, mientras que el desarrollo de las habilidades pedagógicas se ve relegado a un segundo plano, por lo que queda a voluntad de las instituciones brindar oportunidades de formación e incluso bajo la propia responsabilidad de los docentes buscar los mecanismos de actualización y capacitación (de la Cruz y Alamilla, 2018).

En cuanto a la formación requerida, de acuerdo con el tipo de contratación, para los profesores de asignatura generalmente las universidades exigen un nivel académico mínimo de licenciatura y con experiencia laboral comprobable. Por otro lado, para un profesor de tiempo completo el nivel académico solicitado es de maestría, con experiencia en investigación (Universidad Autónoma del Carmen [Unacar], 2009). En ese sentido, los resultados del presente ejercicio demuestran que la relación con el nivel máximo de estudios 


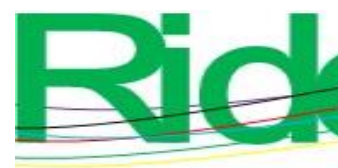

Revista Iberoamericana para la Investigación y el Desarrollo Educativo ISSN 2007-7467

otros. Los resultados de estos ejercicios presuponen un análisis tanto del docente como del área directiva de competencia para diseñar acciones que impacten de manera positiva al programa educativo (Comités Interinstitucionales para la Evaluación de la Educación Superior [Ciees], 2018). En este sentido, pese a que la información analizada no mostró asociación estadísticamente significativa, la percepción de los estudiantes resultó positiva, por consiguiente, representa una oportunidad para mejorar el aprendizaje en el aula y en la calidad del servicio educativo de las universidades (Segovia y Cabello, 2017).

La información recabada muestra que los estudiantes encuestados consideran que sus profesores se apegan a lo establecido en la unidad de aprendizaje, tal como lo describe Segovia y Cabello (2017). Elementos como la puntualidad en clase, administración adecuada del tiempo, contemplar las asistencias, seguimiento de lo establecido en las unidades de aprendizaje y aviso oportuno sobre las notas académicas son valorados adecuadamente por los estudiantes respecto al profesor.

Similar a los reportes de Gómez, y Gómez (2009) y Segovia y Cabello (2017), un referente de la relación docente-estudiante consiste en identificar al profesor como un reflejo de los elementos que dan identidad a la institución, incluyendo ser coherente respecto a su conducción académica, debido a que influye de manera positiva en los estudiantes y en su futuro desempeño profesional.

Las instituciones de educación superior observan un aumento sostenido en los valores de reprobación y deserción, lo que precisa el desarrollo de estudios que ayuden a reconocer los factores que las causan. En consecuencia, en los últimos años se han incrementado las líneas de investigación que abordan estos fenómenos y la manera en la que se relacionan en un contexto educativo. La educación es abordada en los estudios desde diversos paradigmas, sin embargo, el docente ha sido escasamente atendido como sujeto activo del aprendizaje (Pérez et al., 2021). Es por ello por lo que caracterizar los factores docentes y la existencia de una relación con el índice de reprobación son de suma utilidad a la hora de actualizar los programas educativos. 


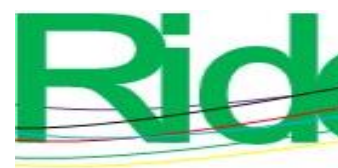

Revista Iberoamericana para la Investigación y el Desarrollo Educativo ISSN $2007-7467$

\section{Conclusiones}

En el ámbito universitario, una de las principales problemáticas que se observa, especialmente durante los primeros semestres, son los elevados índices de reprobación, que son directamente proporcionales a los de la deserción escolar. Se trata de una preocupación común en todas las instituciones de educación superior. La universidad sujeta del presente estudio declara en el modelo educativo como pilar sustancial la formación integral del alumno, reforzado a su vez por diferentes programas institucionales.

La presente investigación subraya el alto grado de complejidad del fenómeno de reprobación y, asimismo, hace hincapié en los factores docentes que están asociados con este. Se trata de una sólida relación entre la formación académica, experiencia profesional y capacidades pedagógicas.

Claramente, la calidad educativa se encuentra relacionada con la calidad del desempeño docente. En esa línea, el diseño de políticas gubernamentales y lineamientos teóricos por parte de las comunidades académicas de reconocido prestigio suele ser insuficiente para incrementar el nivel educativo de un país; es la apropiación crítica y constructiva por parte de los docentes la que permitirá que dichas directrices se constituyan en elementos de valor para alcanzar las metas y los beneficios formativos proyectados.

La presente investigación abona a los hallazgos de otros autores y provee información que permitirá desarrollar acciones que contribuyan a reducir los índices de reprobación y a incrementar las posibilidades de éxito académico de los universitarios.

La mayor limitación encontrada durante la realización de la presente investigación fue la poca cantidad de información disponible para la comparación y análisis de resultados, ya que en otras investigaciones similares realizados por diversos autores existen muchos factores que no se contemplan o que no se asemejan a los factores considerados en el presente estudio, como es el caso de los planes de estudio, pues estos varían mucho entre universidad y universidad. 


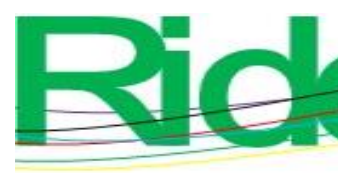

Revista Iberoamericana para la
Investigación y el Desarrollo Educativo ISSN $2007-7467$

\section{Futuras líneas de investigación}

La reprobación es un fenómeno multifactorial. No necesariamente todas las causas son imputables al alumno; por el contrario, existen factores externos relacionados con el rendimiento académico de los estudiantes. Esto hace necesario insistir en la búsqueda de medios o métodos que contribuyan al buen funcionamiento de la educación y así incidir favorablemente en la deserción estudiantil universitaria. Por todo lo anterior, se demanda abrir nuevas líneas que pudieran generar información relevante para las actualizaciones de los programas universitarios y generar estrategias de intervención, evaluación, cobertura e impacto en este nivel.

\section{Referencias}

Anchondo, G., Martínez, R. y Prieto, B. (2020). Impacto en los niveles de deserción y reprobación en los estudiantes usuarios de la estancia infantil de la Universidad Tecnológica de Chihuahua. Revista Gestión Universitaria, 3(7), 9-15.

Campos, B. (2018). Factores pedagógicos que inciden en la reprobación, en voz de los estudiantes normalistas. Interconectando Saberes, 11(6), 127-147. Recuperado de https://doi.org/10.25009/is.v0i6.2584.

Castillo, M., Gamboa, R. y Hidalgo, R. (2020). Factores que influyen en la deserción y reprobación de estudiantes de un curso universitario de matemáticas. Uniciencia, 34(1), 219-245. Recuperado de https://dx.doi.org/10.15359/ru.34-1.13.

Ciro, S. y Reyes, C. M. (2017). Principales causas de reprobación de alumnos de los grupos de quinto semestre grupo seis y ocho de la escuela preparatoria número tres. ConCiencia. Boletín Científico de la Escuela Preparatoria No. 3, 4(7). Recuperado de https://repository.uaeh.edu.mx/revistas/index.php/prepa3/article/view/2079.

Comités Interinstitucionales para la Evaluación de la Educación Superior [Ciees]. (2018). Metodología 2018 para la evaluación y acreditación de programas educativos. Ciudad de México, México: Comités Interinstitucionales para la Evaluación de la Educación Superior.

Consejo Nacional para la Calidad de Programas Educativos en Nutriología [Concapren]. (2016). Informe del proceso de acreditación del programa de Licenciatura en Nutrición de la Universidad Autónoma del Carmen (001). Ciudad de México, México: Consejo Nacional para la Calidad de Programas Educativos en Nutriología. 


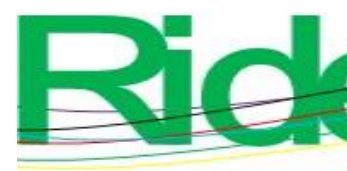

Revista Iberoamericana para la
Investigación y el Desarrollo Educativo
ISSN $2007-7467$

de la Cruz, J. y Alamilla, P. (2018). Experiencias sobre la iniciación a la docencia universitaria en el Profesorado de una institución pública mexicana. Revista Digital de Investigación en Docencia Universitaria, 12(1), 189-212. Recuperado de https://dx.doi.org/10.19083/ridu.12.588.

Díaz, D. y Ruiz, A. (2018). Reprobación escolar en el nivel medio superior y su relación con el autoconcepto en la adolescencia. Revista Latinoamericana de Estudios Educativos, 48(2), 125-142. Recuperado de https://doi.org/10.48102/rlee.2018.48.2.49.

Díaz, K. y Osuna, C. (2017). La percepción y práctica docente en relación al abandono escolar en centros de educación media superior de Baja California. Ponencia presentada en el XIV Congreso Nacional de Investigación Educativa. San Luis Potosí, del 20 al 24 de noviembre de 2017. Recuperado de https://www.comie.org.mx/congreso/memoriaelectronica/v14/doc/0708.pdf.

Dirección General de Educación Superior Universitaria. (2020). Lineamientos del Programa de Carrera Docente en Universidades Públicas Estatales (UPES) fondo extraordinario. Recuperado de https://www.dgesui.ses.sep.gob.mx/documentos/DSA\%20gobmx/Lineamientos\%20 CD\%202020\%20ok.pdf.

Gómez, E. y Gómez, Y. (2009). La evaluación de la docencia basada en la opinión de los alumnos: el perfil docente en la licenciatura en Historia. Ponencia presentada en el X Congreso Nacional de Investigación Educativa. Veracruz, del 21 al 25 de septiembre de 2009.

Lozano, D. y Maldonado, L. (2020). Factores docentes asociados a la reprobación en educación media superior. Dilemas Contemporáneos, 8(3), 1-31.

Martínez, F. (2013). El futuro de la evaluación educativa. Revista Electrónica Sinéctica, (40), 1-11. Recuperado de https://www.redalyc.org/pdf/998/99827467004.pdf.

Mas, O. (2011). El profesor universitario: sus competencias y formación. Profesorado, 15(3), 195-211. Recuperado de https://www.ugr.es/ recfpro/rev153COL1.pdf.

Nieva, J. y Martínez, C. O. (2016). Una nueva mirada sobre la formación docente. Revista Universidad $y \quad$ Sociedad, 8(4), 14-21. Recuperado de http://scielo.sld.cu/scielo.php?script=sci_arttext\&pid=S2218$36202016000400002 \& \operatorname{lng}=\mathrm{es} \& \operatorname{tn} g=\mathrm{es}$.

Ocampo, C. y Cid, B. (2011). Formación, experiencia docente y actitudes de los profesores de infantil y primaria ante la educación escolar de hijos de personas inmigrantes en 

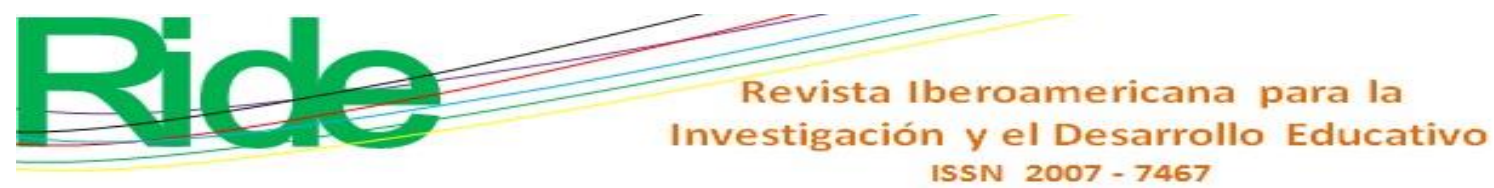

España. Revista de Investigación Educativa, 30(1), 111-130. Recuperado de https://www.redalyc.org/articulo.oa?id=2833/283322861007.

Organización para la Cooperación y el Desarrollo Económicos [OCDE]. (2006). Pisa 2006. Marco de la evaluación. Conocimientos y habilidades en ciencias, matemáticas y lectura. París, Francia: Organización para la Cooperación y el Desarrollo Económicos. Recuperado de https://www.oecd.org/pisa/39732471.pdf.

Pérez, A., Estrada, C. y Estrada, C. (2021). Factores docentes y su asociación con el índice de reprobación en alumnos de nivel superior. Revista RedCA, 3(9), 79-91. Recuperado de https://revistaredca.uaemex.mx/article/view/15100/11739.

Ramírez, L. y Gallur, S. (2017). Percepciones de estudiantes y docentes en torno a la reprobación académica en una institución de educación superior en el contexto fronterizo de Ciudad Juárez. Ponencia presentada en el XIV Congreso Nacional de Investigación Educativa. San Luis Potosí, del 20 al 24 de noviembre de 2017. Recuperado de http://www.comie.org.mx/congreso/memoriaelectronica/v14/doc/0907.pdf.

Sánchez, N. y De Santiago, B. (2017). Factores Relacionados con la reprobación en inglés en educación superior. Conciencia Tecnológica, (54), 27-32. Recuperado de https://www.redalyc.org/jatsRepo/944/94454631008/html/index.html.

Santelices, M. y Valenzuela, F. (2015). Importancia de las características del profesor y de la escuela en la calidad docente: una aproximación desde la teoría de respuesta del ítem. Estudios Pedagógicos (Valdivia), 41(2), 233-254. Recuperado de https://dx.doi.org/10.4067/S0718-07052015000200014.

Sanz, S., Ruiz, C. y Pérez, I. (2014). El profesor universitario y su función docente. Espacio I $+D$, Innovación más Desarrollo, 3(5). Recuperado de https://doi.org/10.31644/IMASD.5.2014.a05.

Secretaría de Educación Pública [SEP]. (2020). Principales cifras del sistema educativo nacional 2019-2020. Ciudad de México, México: Secretaría de Educación Pública. https://www.planeacion.sep.gob.mx/Doc/estadistica_e_indicadores/principales_cifra s/principales_cifras_2019_2020_bolsillo.pdf

Segovia, C. y Cabello, J. (2017). Evaluación docente desde la percepción del estudiante. Lex, 15(19), 409-432. Recuperado de https://doi.org/10.21503/lex.v15i19.1384. 

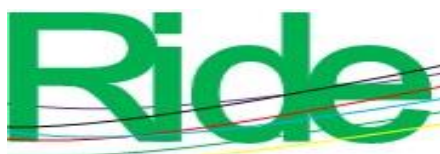

Universidad Autónoma del Carmen [Unacar]. (2009). Reglamento del personal académico. Ciudad del Carmen, México: Universidad Autónoma del Carmen. Recuperado de https://cutt.ly/OlOGD0A.

Valerio, G. y Rodríguez, M. (2017). Perfil del profesor universitario desde la perspectiva del estudiante. Innovación Educativa, 17(74), 109-124. Recuperado de http://www.scielo.org.mx/scielo.php?script=sci_arttext\&pid=S166526732017000200109\&lng=es\&tlng=es.

Villalón, M., Palma, A., Medina, M. y Sillero, J. (2016). Exámenes departamentales como estrategia para reducir la variabilidad en los índices de aprobación. Pistas Educativas, $38(121)$, 233-251.

Recuperado

de http://www.itcelaya.edu.mx/ojs/index.php/pistas/article/view/654. 


\begin{tabular}{|l|l|}
\hline Rol de Contribución & $\begin{array}{c}\text { Revista Ineroamericana para la } \\
\text { Inst el Desarrollo Educativo }-7467\end{array}$ \\
\hline Conceptualización & Autor (es) \\
\hline Metodología & Ángel Esteban Torres Zapata \\
\hline Software & Carmen Cecilia Lara-Gamboa \\
\hline Validación & César Uziel Estrada-Reyes \\
\hline Análisis Formal & Ángel Esteban Torres Zapata \\
\hline Investigación & César Uziel Estrada-Reyes. \\
\hline Recursos & Carmen Cecilia Lara-Gamboa \\
\hline Curación de datos & Ángel Esteban Torres Zapata \\
\hline $\begin{array}{l}\text { Escritura - Preparación del } \\
\text { borrador original }\end{array}$ & César Uziel Estrada-Reyes \\
\hline $\begin{array}{l}\text { Escritura - Revisión y } \\
\text { edición }\end{array}$ & Alejandra Karina Pérez-Jaimes \\
\hline Visualización & Ángel Esteban Torres Zapata \\
\hline Supervisión & Ángel Esteban Torres Zapata \\
\hline Administración de Proyectos & Carmen Cecilia Lara-Gamboa \\
\hline Adquisición de fondos & $\begin{array}{l}\text { Ángel Esteban Torres Zapata, Alejandra Karina Pérez-Jaimes, } \\
\text { César Uziel Estrada-Reyes y Carmen Cecilia Lara-Gamboa }\end{array}$ \\
\hline
\end{tabular}

